\title{
Detecção automática e dinâmica de Estilos de Aprendizagem para Sistemas Adaptativos Inteligentes para a Educação utilizando a meta-heurística Vitis Vinífera
}

\author{
Hilton Leslie ${ }^{1}$,Alessandro Vivas ${ }^{1}$, Luciana Assis ${ }^{1}$, \\ Cristiano Pitangui ${ }^{2}$, Arthur Machado ${ }^{1}$, Fabiano Dorça ${ }^{3}$ \\ ${ }^{1}$ DECOM - UFVJM \\ Diamantina - MG \\ ${ }^{2}$ DTECH - UFSJ \\ Ouro Branco, MG. \\ ${ }^{3}$ FACOM - UFU \\ Uberlândia, MG. \\ \{hiltontur, alessandro.vivas, lupassis\}@gmail.com \\ \{pitangui.cristiano, arthurmfalmeida, fabianodorca\}@gmail.com
}

\begin{abstract}
The present work adresses the problem to detect students' cognitive traits in order to customize Virtual Learning Enviroments. In this sense, it applies the Vits Vinifera meta-heuristic to detect the student's Learning Style for the selection of learning objects in order to improve the teaching and learning processes. The proposed approach is evaluated in relation to the state of the art that uses a Genetic Algorithm for the identification of the Learning Style. Experimental results indicate that the proposed approach is superior in relation to the Genetic Algorithm considering the detections erros.
\end{abstract}

Resumo. O presente trabalho aborda o problema de detecção de traços cognitivos de estudantes para customização de Ambientes Virtuais de Aprendizagem. Neste sentido, aplica-se a meta-heurística Vits Vinífera para a detecção do Estilo de Aprendizagem do aluno para futura seleção de objetos de aprendizagem de forma a aprimorar o processo de ensino-aprendizagem. A abordagem proposta é avaliada em relação ao estado da arte que utiliza um Algoritmo Genético para a identificação do Estilo de Aprendizagem. Resultados experimentais apontam que a abordagem proposta é superior em relação ao estado da arte considerando o número de erros de detecção cometidos.

\section{Introdução}

Um dos maiores desafios de plataformas de ensino virtuais é se opor ao paradigma one size fits all, onde o mesmo conteúdo é oferecido a todos os estudantes da mesma maneira. A investigação profunda deste problema deu origem aos chamados Sistemas Adaptativos e Inteligentes para Educação (SAIE).

Esses sistemas educacionais se caraterizam por adaptarem o conteúdo apresentado e o suporte à navegação, de acordo com as características individuais dos estudantes. Dorça (2012) afirma que a detecção fundamentada em uma abordagem probabilística para 
VII Congresso Brasileiro de Informática na Educação (CBIE 2018)

Anais do XXIX Simpósio Brasileiro de Informática na Educação (SBIE 2018)

modelagem do estudante é um importante problema, visto a necessidade desses modelos lidarem com informações incompletas ou incertas sobre o estudante [Dorça 2012].

A modelagem do estudante baseada em Estilos de Aprendizagem (EA), por meio do perfil cognitivo do estudante, serve como base para a estruturação do ensino personalizado nesses sistemas. Existem diversos algoritmos e meta-heurísticas que modelam processos estocásticos e que podem ser aplicados no auxílio à personalização dos conteúdos nos SAIE. Neste sentido, a escolha do método utilizado influencia grandemente o sucesso destes sistemas.

O presente trabalho apresenta uma abordagem baseada na meta-heurística Vitis Vinifera (MHV) [Oliveira et al. 2018, Oliveira et al. 2017] para a detecção dinâmica e automática de Estilos de Aprendizagem para SAIE. A abordagem proposta é avaliada em relação ao estado da arte [Yannibelli et al. 2006] que utiliza um Algoritmo Genético (AG) [Goldberg and Holland 1988, Holland 1992] para a detecção de EA de alunos.

Este trabalho se organiza como segue. A seção 2 apresenta o referencial teórico e os principais trabalhos relacionados a esta pesquisa. A seção 3 apresenta a modelagem do problema de detecção de EA segundo [Yannibelli et al. 2006]. A seção 4 apresenta a abordagem proposta para a detecção automática e dinâmica de EA. A seção 5 apresenta os resultados experimentais obtidos. Finalmente, a seção 6 conclui o trabalho e apresenta perspectivas de trabalhos futuros.

\section{Referêncial Teórico}

\subsection{Estilos de Aprendizagem}

Este trabalho adota o modelo de Estilos de Aprendizagem proposto por Felder e Silverman [Felder et al. 1988] conhecido como Felder and Silverman Learning Style Model (FSLSM). O modelo foi escolhido uma vez que ele é o mais utilizado na literatura correlata [Zaina et al. 2012] e ainda apresenta natureza dinâmica representada por meio de distribuição de probabilidades.

O FSLSM é composto por 4 dimensões a saber, Percepção, Entrada, Processamento, e Compreensão. Inicialmente, cada dimensão assumia um de dois valores possíveis, assim, este modelo considerava a existência de 16 possíveis Estilos de Aprendizagem $\left(2^{4}=16\right)$.

Para explorar todas as possibilidades de cada dimensão, Felder desenvolveu o Index of Learning Styles Questionnaire [Felder et al. 2002] (ILS). Neste sentido, cada dimensão passou a ser classificada com valores extremos, médios ou neutros, incorporando, ao modelo, alunos neutros, e alunos com uma tendência a um estilo, mas que possuem características de outro.

Com essas alterações, é possível atribuir um de 5 valores a cada dimensão. Dessa forma, o modelo considera a existência de $625\left(5^{4}=625\right) \mathrm{EA}$, um universo muito mais abrangente do que o considerado inicialmente.

\subsection{Trabalhos Relacionados}

No ano de 2012 foi proposto o primeiro trabalho para detecção de Estilos de Aprendizagem utilizando Cadeias de Markov e Aprendizado por Reforço [Dorça 2012]. 
VII Congresso Brasileiro de Informática na Educação (CBIE 2018)

Anais do XXIX Simpósio Brasileiro de Informática na Educação (SBIE 2018)

[Gonçalves et al. 2016] alterou a política de aplicação de reforços e obteve uma melhoria de $6.1 \%$ no desempenho avaliativo e uma redução média de $68.3 \%$ nos problemas de aprendizagem.

[Rodrigues et al. 2016] apresentou melhorias no Modelo Pedagógico do sistema apresentado em [Dorça 2012], por meio da utilização de Lógica Fuzzy no processo de seleção de Combinação de Estilos de Aprendizagem (CEA). Estas melhorias aumentaram a velocidade de convergência do sistema, mas implicaram em um determinismo no processo de detecção do EA.

Em 2017, vários trabalhos abordaram o problema da detecção determinística de EA. [Salazar et al. 2017] propôs uma abordagem baseada em Redes Bayesianas. [Ribeiro et al. 2017] propôs um algoritmo baseado em Média Móvel Exponencialmente Ponderada. [Silva et al. 2017] propôs uma técnica baseada em Dynamic Scripting para acelerar o processo de detecção do EA .

Em 2018 foram propostas duas abordagens para redução dos erros de detecção de EA. A primeira abordagem foi proposta por [Silva et al. 2018] que aprimorou seu método apresentado em 2017, e [Falci et al. 2018] que combinou duas abordagens para o aumento da velocidade de detecção de EA .

\subsection{A Meta-heurística Vitis Vinifera}

A meta-heurística Vitis Vinifera (MHV) [Oliveira et al. 2017, Oliveira et al. 2018] classifica-se como uma meta-heurística bio-inspirada que tem como inspiração o comportamento e crescimento da videira.

Assim como o crescimento da planta é influenciado por fatores iniciais ao seu plantio e pelas influências exercidas durante seu desenvolvimento, modelou-se a MHV de forma a abranger os principais fatores e influencias que determinam o desenvolvimento da planta.

De forma geral, a MHV segue os passos descritos a seguir. Uma descrição mais detalhada pode ser encontrada em [Oliveira et al. 2017, Oliveira et al. 2018].

1. Geram-se $n$ (parâmetro de entrada do algoritmo) soluções iniciais aleatoriamente. Este conjunto de soluções é chamado de Videira Inicial (VI);

2. Calculam-se as qualidades das $n$ soluções em VI de acordo com a função objetivo do problema abordado;

3. Geram-se $n$ novas soluções por meio da modificação das soluções em VI. Este novo conjunto de soluções é chamado de Videira Temporária (VT).

As modificações nas soluções em VI ocorrem em função da temperatura do ciclo (iteração) atual do algoritmo. Basicamente, em ciclos iniciais, as soluções sofrem mais alterações que em ciclos tardios do algoritmo;

4. Gera-se uma nova videira, denominada de Videira Selecionada (VS), que possui $n$ soluções. Esta videira é criada pela seleção probabilística (de acordo com a qualidade) das soluções em VI e VT.

Este procedimento reflete o processo natural de desenvolvimento da videira em que galhos mais promissores recebem mais seiva e tendem a se desenvolverem ainda mais, enquanto galhos mais fracos tendem a receber menos seiva e, portanto, propensam-se a serem desprezados; 
VII Congresso Brasileiro de Informática na Educação (CBIE 2018)

Anais do XXIX Simpósio Brasileiro de Informática na Educação (SBIE 2018)

5. Atualizam-se as soluções da videira VS por meio da inserção de partes de uma solução selecionada em outra. Para este fim, selecionam-se aleatoriamente duas soluções em VS, a saber $s$ ' e $s$ ". Caso a qualidade de $s$ ' seja melhor que a de $s$ ", parte da estrutura de $s$ ' será inserida em $s$ ”. A quantidade de material inserido em $s$ " é determinado proporcionalmente pela diferença entre as qualidades das soluções de s' e $s$ ". Assim, caso as qualidades de $s$ ' e $s$ ” sejam semelhantes, pouco da estrutura de $s$ ' será inserida em $s$ ". Em contrapartida, caso a diferença entre as qualidades seja elevada, mais da estrutura de $s$ ' será inserida em $s$ ”.

Este processo origina uma nova solução denominada $s$ "' (formada pela inserção de parte da estrutura de $s^{\prime}$ em $s$ ”). Em seguida, a qualidade da solução $s$ ”' é calculada e comparada à qualidade da solução de $s$ ". Caso $s$ "' seja melhor que $s$ ”, a solução $s$ ” é substituída pela solução $s$ "' em VS. Caso contrário, mantem-se a solução $s$ " em VS.

O processo descrito é repetido $n$ vezes para a atualização da videira VS. Ao fim do processo descrito, atribui-se a videira VS atualizada à videira VI e o algoritmo retorna ao passo 2. Os passos 1 a 5 são repetidos $N$ vezes, em que $N$ é um dos parâmetros de entrada do algoritmo. Ao fim da execução do algoritmo, retorna-se a melhor solução em VI como solução do problema abordado.

É importante destacar que o passo 5 tem a função de emular a técnica de enxerto em videiras, onde partes de uma videira promissora (que se mostra bem adaptada ao ambiente) são inseridas em outra.

\subsection{Algoritmos Genéticos}

Os Algoritmos Genéticos (AG) [Goldberg and Holland 1988] [Holland 1992] são metaheurísticas inspiradas na teoria da evolução por seleção natural. Como consequência, fazem uso de procedimentos iterativos que simulam o processo de evolução, e de operadores de busca baseados na recombinação (crossover) e mutação gênicas.

Neste sentido, a cada iteração (geração) um novo conjunto de soluções (população) é gerado por meio da seleção de soluções (indivíduos) mais qualificadas (adaptadas) e pela aplicação dos operadores de busca de recombinação e mutação. Como resultado, a tendência é que, a cada iteração, gerem-se soluções cada vez melhores e mais próximas da solução ótima.

\section{Modelagem do Problema}

O presente trabalho aplica a MHV ao problema de detecção automática de EA no contexto da abordagem proposta por Yannibelli et al. (2006) [Yannibelli et al. 2006]. O referido trabalho utiliza um AG para detectar o EA de aprendizes por meio de suas iterações em um AVA considerando o FSLSM. Neste sentido, foram consideradas as seguintes ações dos alunos no AVA, a saber: 1) participação em fóruns; 2) participação em chats; 3) leitura do material; 4) acesso a exemplos; 5) realização de exercícios; 6) tempo de entrega de exames; 7) correção de exercícios; 8) mudanças de respostas; 9) maneira pela qual os aprendizes acessam o conteúdo (sequencialmente ou globalmente).

As ações 1) e 2) estão relacionadas à dimensão de Processamento, indicando a preferência dos alunos em trabalharem sozinhos (Reflexivos) ou em grupos (Ativos). As ações 3), 4), 5), 6), 7), e 8) são referentes à dimensão de Percepção, indicando se os 
VII Congresso Brasileiro de Informática na Educação (CBIE 2018)

Anais do XXIX Simpósio Brasileiro de Informática na Educação (SBIE 2018)

aprendizes são Sensitivos ou Intuitivos. Por sua vez, a ação 9) pertence à dimensão de Compreensão, revelando um conduta Sequencial ou Global de pensamento. A dimensão de Entrada não é levada em consideração no estudo realizado em [Yannibelli et al. 2006], devido a ausência de vídeos e demonstrações no AVA considerado.

A autora atribuiu valores numéricos que mensuram, de forma geral, o índice de participação dos alunos em cada um dos 9 itens apresentados. A codificação adotada é apresentada a seguir.

1. Participação em fóruns: o valor 0 reflete a não participação, 1 , se apenas lê as mensagens, 2, se lê as mensagens no fórum e as responde, e, 3, se envia mensagens que iniciem discussões;

2. Participação em chat: o valor 0 reflete a não participação, 1 , se apenas lê as mensagens, e, 2, se lê as mensagens no chat e as responde;

3. Leitura do material: o valor 0 expressa apenas a leitura do material teórico, 1 , se lê todo material teórico e parte do prático, 2, se lê o material teórico e também o prático, 3, se lê todo material prático e parte do teórico, e, 4, se apenas lê o material prático;

4. Acesso aos exemplos e realização de exercícios: o valor 0 reflete índice de acesso menor que $10 \%$, 1 , entre $10 \%$ e $30 \%, 2$, entre $31 \%$ e $50 \%, 3$, entre $51 \%$ e $70 \%$ e, 4, mais que $70 \%$;

5. Realização de exercícios: o valor 0 reflete índice de acesso menor que $10 \%, 1$, entre $10 \%$ e $30 \%, 2$, entre $31 \%$ e $50 \%$, 3, entre $51 \%$ e $70 \%$ e, 4 , mais que $70 \%$;

6. Tempo de entrega dos exames (tempo utilizado pelo aluno para finalizar um exame): o valor 0 reflete a utilização de um tempo menor que $40 \%$ do tempo fornecido, 1 , entre $41 \%$ e $50 \%$, 2 , entre $51 \%$ e $60 \%$, 3, entre $61 \%$ e $70 \%$ e, 4 , mais que $70 \%$ do tempo fornecido;

7. Tempo utilizado para correção de exames: o valor 0 reflete a utilização de menos que $5 \%$ do tempo fornecido, 1 , entre $5 \%$ e $10 \%$, 2, entre $11 \%$ e $15 \%$, 3, entre $16 \%$ e $20 \%$, 4, mais que $20 \%$ do tempo fornecido;

8. Mudanças de respostas dadas: o valor 0 reflete mudança de menos de $10 \%$ das questões, 1 , entre $10 \%$ e $30 \%$, 2 , entre $31 \%$ e $50 \%$, 3, entre $51 \%$ e $70 \%$ e, 4 , mais de $70 \%$ das respostas dadas;

9. Tipo de navegação: o valor 0 reflete uma navegação totalmente sequencial, 1 , para maior parte sequencial, 2, equilíbrio em sequencial e global, 3, maior parte global, e, 4 , totalmente global.

O trabalho relaciona ainda os valores numéricos atribuídos às ações dos alunos dentro de uma das três dimensões consideradas. Tais relações são dadas pela soma dos valores numéricos e representam as tendências dos alunos a se comportarem de acordo com uma das categorias da dimensão analisada. A seguir, apresentam-se tais relações categorizadas pelas dimensões consideradas.

- Dimensão de Processamento: como apresentado, esta dimensão se relaciona a ações realizadas no fórum e participação em chats. O valor é dado pela soma dos valores numéricos atribuídos às ações consideradas por esta dimensão. Se o resultado do somatório for igual a 0 , representa um aluno extremamente passivo, igual a 1, médio passivo, entre 2 e 3, neutro, igual a 4, médio ativo, e, igual a 5, extremamente ativo. 
VII Congresso Brasileiro de Informática na Educação (CBIE 2018)

Anais do XXIX Simpósio Brasileiro de Informática na Educação (SBIE 2018)

- Dimensão Percepção: como apresentado, esta dimensão se relaciona a ações de leitura de material, acesso a exemplos, realização de exercícios e tempo de entrega dos mesmos, tempo utilizado para correção de exames, e mudanças nas respostas dadas. Neste sentido, o valor da dimensão é dado pela soma dos valores numéricos atribuídos às ações consideradas por esta dimensão. Se o resultado do somatório estiver entre 0 e 4 , extremamente intuitivo, entre 4 e 8 , médio intuitivo, entre 8 e 16, neutro, entre 16 e 20, médio sensitivo, e, entre 20 e 24 , extremamente sensitivo.

- Dimensão Compreensão: como apresentado, esta dimensão se relaciona ao tipo de navegação utilizada. Neste sentido, o valor da dimensão é dado pelo valor numérico atribuído ao padrão de navegação do aprendiz. Se o valor do somatório for igual a 0 , aponta um comportamento extremamente sequencial, igual a 1, médio sequencial, igual a 2, neutro, igual a 3, médio global, e igual a 4, extremamente global.

Como exemplo, um aluno que tenha o comportamento (vide sequencialmente os itens de 1 a 9 apresentados): 2, lê as mensagens no fórum e as responde; 4, apenas lê o material prático; 2 , observa entre $31 \%$ e $50 \%$ dos exemplos apresentados; 4 , faz mais de $70 \%$ dos exercícios apresentados; 3 , usa entre $61 \%$ e $70 \%$ do tempo fornecido; 4 , usa mais de $20 \%$ do tempo fornecido; 3 , modifica entre $51 \%$ e $70 \%$ das respostas dadas e, 1 , verifica a maioria dos itens de forma sequencial e o restante de modo global. Tal aluno receberia como resultado para a dimensão Processamento, nota 2. Na dimensão Percepção, sua nota seria $20(4+2+4+3+4+3)$. Por fim, na dimensão Compreensão, receberia nota 1. Este aluno seria, portanto, categorizado na dimensão de Processamento como neutro, na dimensão de Percepção como médio-sensitivo, e na dimensão Compreensão como médio-sequencial.

\section{Abordagem Proposta}

Adota-se a mesma representação da solução utilizada em [Yannibelli et al. 2006]. Neste sentido, as soluções na videira representam as ações do estudante durante uma seção de aprendizagem. Seguindo esse raciocínio, todas as 9 ações realizadas pelo aluno são representadas em um vetor que corresponderá a uma possível solução do problema.

Tal como [Yannibelli et al. 2006], a geração inicial das soluções é realizada de forma aleatória de maneira a cobrir diferentes pontos do espaço de busca. Ainda, e de acordo com o referido trabalho, a qualidade de uma solução $i$, dada por $q_{i}$, reflete a distância do EA detectado (por meio conjunto de ações realizada pelo aprendiz em $i$ ) em relação ao EA real (que almeja-se detectar). Assim, a função de qualidade baseia-se no grupo de ações $A=\left\{a_{1}, a_{2}, \ldots, a_{9}\right\}$ explicadas descritas anteriormente.

A MHV utilizou o método de torneio ternário como método de seleção. Assim, durante a seleção das soluções (em VI e VT) para a geração da videira VS, selecionamse 3 soluções aleatoriamente de VI e VT, e a que possuir maior qualidade é considerada a vencedora do torneio e, portanto, escolhida para compor a videira VS. Como apontado anteriormente, este processo é repetido $n$ vezes a fim de que a videira VS possua $n$ soluções. 
VII Congresso Brasileiro de Informática na Educação (CBIE 2018)

Anais do XXIX Simpósio Brasileiro de Informática na Educação (SBIE 2018)

\section{Resultados Experimentais}

\subsection{Metodologia Experimental}

A abordagem proposta foi avaliada em relação a [Yannibelli et al. 2006] que utiliza um AG no processo de detecção dos EA. Realizaram-se 30 testes independentes em cada abordagem (MHV e AG). Cada um dos testes tem como objetivo detectar o EA de 30 alunos. Assim, no total, cada uma das abordagens foi submetida à 900 tentativas de detecção de EA.

O AG avaliou no total 100.000 soluções, utilizou taxa de recombinação de $100 \%$, taxa de mutação de 1\%, e torneio ternário como método de seleção. Por sua vez, a MHV, de modo a realizar uma comparação justa, também avaliou 100.000 soluções, utilizou torneio ternário como método de seleção, e todos os outros parâmetros assumiram o valores default conforme apresentados em [Oliveira et al. 2017]

Utilizou-se a escala Likert para para mensurar o nível de precisão na detecção do EA. Cada dimensão do FSLSM pode receber um valor de 1 a 5 (veja seção 2.1), onde 1 representa a influência de apenas uma dimensão, e, 5, a influência apenas da dimensão oposta. Neste sentido, considerando a dimensão Processamento, o valor 1 representa um aprendiz passivo e, o valor 5, ativo. O valor 3 representa um valor neutro, que pode ser interpretado como um meio termo entre as influências de ambas as dimensões. Por sua vez, os valores 2 e 4 representam influências com certas tendências. Assim, ainda considerando a dimensão Processamento, tem-se que o valor 2 representa um aluno passivo tendendo a neutralidade, enquanto um valor 4 representa um aluno medianamente ativo tendendo ao totalmente ativo.

Para calcular o nível de precisão de uma detecção (solução), calcula-se a distância euclidiana entre o EA detectado e o EA real. Claramente, quando menor a distância entre o EA detectado e EA real, maior é a precisão da detecção. Uma detecção exata ocorre quando a distância entre o EA detectado e o EA real assume o valor 0.

\subsection{Resultados}

A Figura 1 apresenta, para cada abordagem, a média das distâncias entre o EA detectado e o EA real para as 900 detecções realizadas. Observa-se que a MHV incorreu numa menor quantidade de erros, 33, em relação ao AG, 237.

A Tabela 1 apresenta o valor mínimo, primeiro quartil, mediana, média, terceiro quartil, e valor máximo, considerando a distância, respectivamente para a MHV e o AG. Tais valores tornam ainda mais clara a diferença entre os resultados obtidos, visto que até 75\% dos resultados (terceiro quartil), a MHV detecta exatamente o EA real dos aprendizes (diferença entre o EA detectado e EA real é igual a 0).

Tabela 1. Sumário dos resultados obtidos para a MHV e o AG

\begin{tabular}{|c|c|c|c|c|c|c|}
\hline Abordagem & Min & Q1 & Mediana & Mean & Q3 & Max \\
\hline MHV & 0.00 & 0.00 & 0.00 & 0.1375 & 0 & 4 \\
\hline AG & 0.00 & 0.00 & 1.00 & 0.9875 & 1 & 6 \\
\hline
\end{tabular}

Ademais, o desvio padrão obtido pela MHV é de 0.56, e o do AG é de 1.2, o que deixa claro que a MHV possui menor variabilidade em seus resultados, apresentando-se, portanto, como uma técninca mais estável que o AG. 
VII Congresso Brasileiro de Informática na Educação (CBIE 2018)

Anais do XXIX Simpósio Brasileiro de Informática na Educação (SBIE 2018)

Distância Total dos EA's Detectados para os Reais

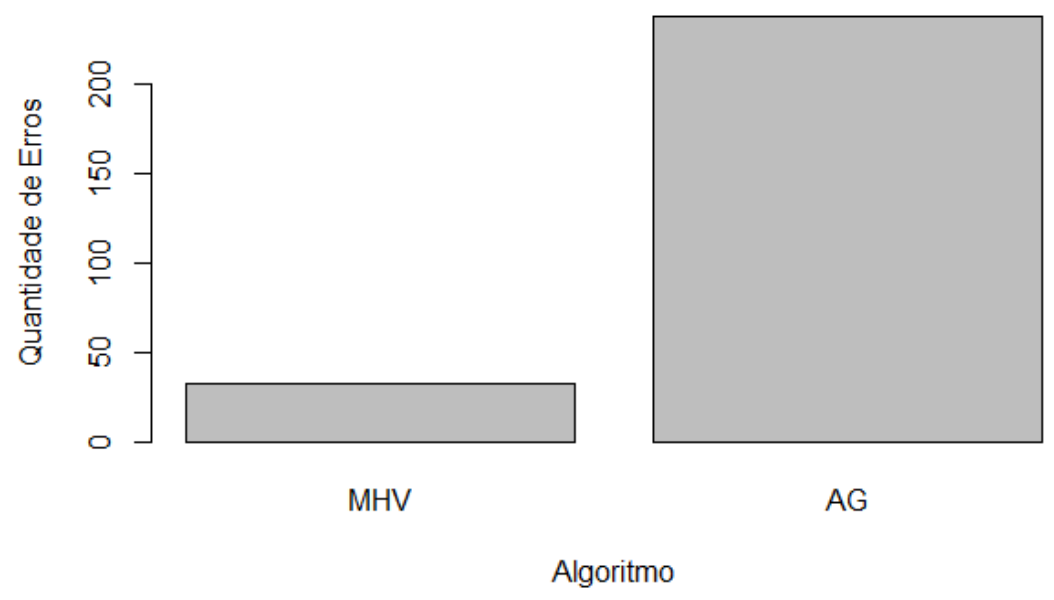

Figura 1. Distância Total dos EA Detectados para os Reais

A figura 2 apresenta os histogramas relativos às distâncias entre os EAs detectados e o EAs reais para ambas as abordagens consideradas. A MHV possui uma frequência muito maior de zeros que o AG. Já o AG possui grande incidência de distâncias com valor 1, e mais de 100 incidências de valores de distâncias iguais ou superiores a 2.

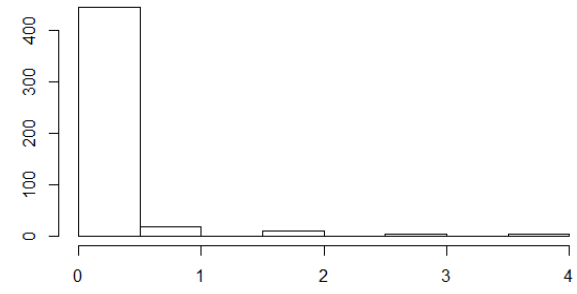

(a) MHV

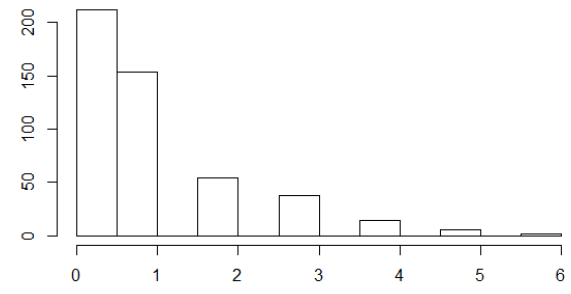

(b) AG

Figura 2. Histogramas

O boxplot apresentado na figura 3 refelete a dispersão dos valores em torno da média, que, para a MHV, é praticamente 0 , e, para o AG, aproxima-se de 1 . Nos resultados obtidos pela MHV, pode-se observar que valores acima de 1 já são considerados outliers, enquanto no AG isso só acontece para valores iguais ou superiores a 3.

A hipótese nula do teste estatístico utilizado (Shapiro-Wilk) é que a população está normalmente distribuída. Sendo assim, para $p<0.05$, a hipótese nula é rejeitada, ou seja, os dados não possuem distribuição normal. Para a MHV, tem-se $\mathrm{w}=0.2666 \mathrm{com}$ $p-$ value $<2.2 e^{-16}$, e para o $\mathrm{AG}$, tem-se $\mathrm{w}=0.782$ com $p-$ value $<2.2 e^{-16}$. Neste sentido, os testes de normalidade dos dois algoritmos possuem $\mathrm{p}$-valor muito inferiores a 0.05, deixando claro a não-normalidade dos dados.

Uma vez que o conjunto de dados testados não são normalmente distribuídos, não são paramétricos, e são pareados, utilizou-se o teste de hipóteses de Wilcoxon com a finalidade de comparar as duas amostras para avaliar se as distribuições médias populacionais possuem diferenças relevantes. Os resultados são apresentados na figura 4 
VII Congresso Brasileiro de Informática na Educação (CBIE 2018)

Anais do XXIX Simpósio Brasileiro de Informática na Educação (SBIE 2018)

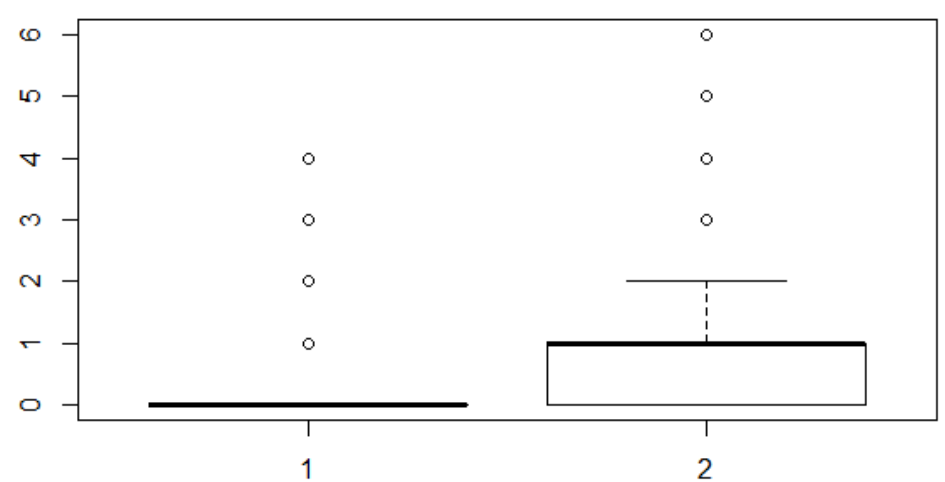

Figura 3. Boxplot comparativo dos dados

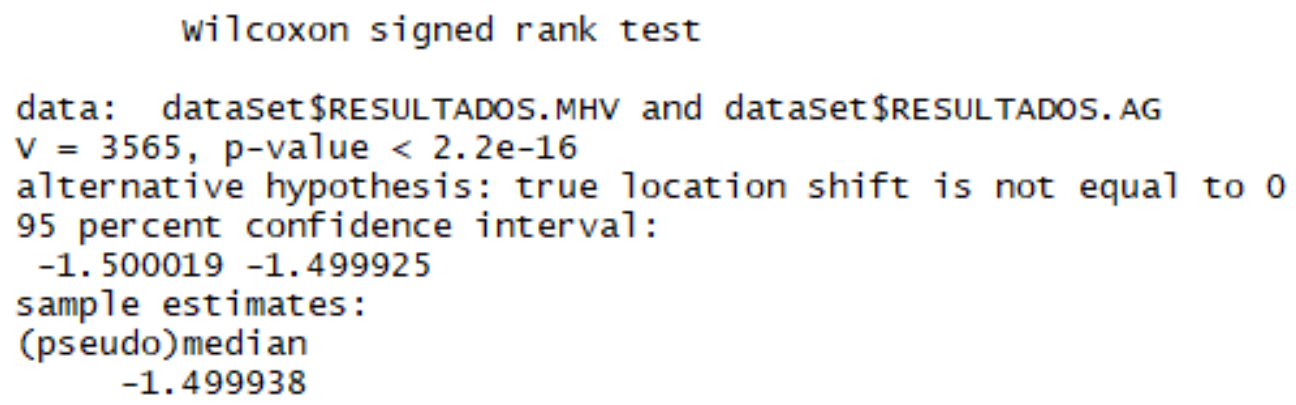

Figura 4. Teste de Hipóteses (Wilcoxon)

O resultado mostra que há diferença significativa, sendo os resultados obtidos pela MHV substancialmente melhores do que os obtidos pelo AG.

\section{Conclusões}

Os resultados obtidos pela MHV mostram-se superiores em relação a abordagem considerada estado da arte que utiliza um AG para a detecção de EA. A melhoria significativa na detecção possibilita a descoberta do EA do usuário de forma mais precisa, tornando mais assertiva a adaptatividade dos sistemas que tem por objetivo ofertarem conteúdos personalizados à estudantes.

Um possível futuro trabalho seria a indicação de objetos de aprendizagem adequados a partir do EA detectado por meio das interações do estudante com o sistema.

\section{Referências}

Dorça, F. A. (2012). Fabiano azevedo dorça uma abordagem estocástica baseada em aprendizagem por reforço para modelagem automática e dinâmica de estilos de aprendizagem de estudantes em sistemas adaptativos e inteligentes para a educação a distância. Universidade Federal de Uberlândia, page 208.

Falci, S., Vivas, A., Assis, L., Carvalho, L., and Dorça, F. (2018). Detecção de estilos de aprendizagem utilizando lógica fuzzy e categorização de reforços. CIET:EnPED. 
VII Congresso Brasileiro de Informática na Educação (CBIE 2018)

Anais do XXIX Simpósio Brasileiro de Informática na Educação (SBIE 2018)

Felder, R. M., Silverman, L. K., et al. (1988). Learning and teaching styles in engineering education. Engineering education, 78(7):674-681.

Felder, S. B. a., Carolina, N., and , R. M. (2002). Index of Learning Styles Questionnaire. Learning, pages $1-5$.

Goldberg, D. E. and Holland, J. H. (1988). Genetic algorithms and machine learning. Machine Learning, 3(2):95-99.

Gonçalves, A. V., Vivas, A., Assis, L., Pitangui, C., and Dorça, F. (2016). Avanços na modelagem automática e dinâmica de estilos de aprendizagem de estudantes em sistemas adaptativos e inteligentes para educação: uma análise experimental. In Simpósio Brasileiro de Informática na Euducação, page 1006.

Holland, J. H. (1992). Genetic algorithms. Scientific american, 267(1):66-73.

Oliveira, H. L., Vivas, A., Assis, L., Pitangui, a., and Lana, L. (2018). MHV - uma nova meta-heurística inspirada no comportamento da Vitis Vinífera, volume 1, chapter 6, pages 76-87. Editora Poison.

Oliveira, H. L., Vivas, A., Assis, L., Pitangui, C., and Lana, L. (2017). Uma Nova MetaHeurística Inspirada no Comportamento da Vitis Vinifera. In XXIV Simpósio de Engenharia de Produção, Marília.

Ribeiro, P., Assis, L., Vivas, A., and Pitangui, C. (2017). Detecção de Estilos de Aprendizagem utilizando Média Móvel Exponencialmente Ponderada. In Simpósio Brasileiro de Informática na Educação, page 1247, Recife.

Rodrigues, L. H. S., Assis, L., Vivas, A., Pitangui, C., and Falci, S. (2016). Análise comparativa de novas abordagens para modelagem automática e dinâmica de estilos de aprendizagem em sistemas adaptativos e inteligentes para educação. In Simpósio Brasileiro de Informática na Educação, page 1076.

Salazar, L., Assis, L., Vivas, A., Pitangui, C., and Falci, S. (2017). Detecção de Estilos de Aprendizagem em Ambientes Virtuais de Aprendizagem Utilizando Redes Bayesianas. In Simpósio Brasileiro de Informática na Educação, page 1317, Recife.

Silva, J., Pitangui, C., Vivas, A., and Assis, L. (2018). Uma nova abordagem para sistemas adaptativos e inteligentes utilizando dynamic scripting. Revista Eletrônica ArgentinaBrasil de Tecnologias da Informação e da Comunicação, 1(8).

Silva, J. C., Pitangui, C., Assis, L., and Vivas, A. (2017). Detecção Automática e Dinâmica de Estilos de Aprendizagem em Sistemas Adaptativos e Inteligentes utilizando Dynamic Scripting. In Simpósio Brasileiro de Informática na Educação, page 1327, Recife.

Yannibelli, V., Godoy, D., and Amandi, A. (2006). A genetic algorithm approach to recognise students learning styles. Interactive Learning Environments, 14(1):55-78.

Zaina, L. A. M., Bressan, G., Cardieri, M. A. A. C., and Rodrigues Júnior, J. F. (2012). e-LORS: Uma Abordagem para Recomendação de Objetos de Aprendizagem. Revista Brasileira de Informática na Educação, 20(1):4. 